\title{
Особенности когерентной спиновой динамики двумерного электронного газа в режиме холловского ферромагнетика
}

\author{
(C) А.В. Ларионов ${ }^{1}$, Э. Степанец-Хуссейн ${ }^{1.2}$, Л.В. Кулик ${ }^{1,2}$ \\ ${ }^{1}$ Институт фризики твердого тела Российской академии наук, \\ 142432 Черноголовка, Россия \\ ${ }^{2}$ Национальный исследовательский университет „Высшая школа экономики“, \\ 141700 Москва, Россия \\ E-mail: larionov@issp.ac.ru
}

Поступила в Редакцию 15 апреля 2020 г.

В окончательной редакции 21 апреля 2020 г.

Принята к публикации 21 апреля 2020 г.

С помощью методики время-разрешенного керровского вращения исследована спин-деполяризованная электронная система на факторе заполнения $v=1$ в квантовой яме GaAs. При температурах выше $5 \mathrm{~K}$ обнаружен переход из нового спин-коррелированного состояния в состояние с низкой спиновой жесткостью, характерной для одночастичной электронной системы. Выделен нелинейный вклад в затухание ларморовских осцилляций, возникающий при низких температурах, когда спин-спиновые корреляции определяют основное состояние двумерной электронной системы. Сделаны оценки параметров флуктуирующего магнитного поля, действующего на отдельные электронные спины.

Ключевые слова: холловский ферромагнетик, спиновая жесткость, керровское вращение, ларморовские осцилляции.

DOI: $10.21883 /$ FTP.2020.09.49843.39

\section{1. Введение}

В последнее время большое внимание научной общественности уделяется областям технологических приложений, использующих манипулирование спиновой степенью свободы. В случае двумерных электронных систем (2ДЭС) в магнитном поле квантово-холловский ферромагнетик до сих пор являлся наиболее изученным спиновым объектом. Открытым вопросом на настоящий момент является описание основного состояния холловского ферромагнетика при конечных температурах, когда происходит сильная спиновая деполяризация. Важный шаг в изучении свойств спиндеполяризованных состояний был связан с попыткой адресоваться к спиновой жесткости, определяемой величиной обменного взаимодействия между соседними спинами, с помощью методики время-разрешенного керровского вращения. Идея этой экспериментальной методики состоит в импульсном отклонении полного спина электронной системы от его равновесного направления, параллельного магнитному полю, на малый угол и последующем исследовании динамики спиновой прецессии вокруг направления внешнего магнитного поля.

Ранее в работе [1] была предложена модель, описывающая время сбоя фазы когерентной прецессии электронов вследствие пространственных флуктуаций эффективного $g$-фактора электронов. При низких гелиевых температурах существенный вклад в эффективное магнитное поле, приводящее к флуктуациям $g$-фактора электронов, также вносят ядерные спины. Два эти вклада можно объединить в один, считая, что на электронные спины действует эффективное флуктуирующее магнитное поле, определяемое локальным эффективным $g$-фактором отдельного электрона и контактным взаимодействием этого электрона с локальным полем ядерной подсистемы. В пренебрежении обменным взаимодействием между электронами коллективная прецессия 2ДЭС распадается на локальные прецессии отдельных спинов с индивидуальной частотой Лармора, определяемой локальным значением флуктуирующего магнитного поля. В свою очередь спиновая жесткость, связанная с многочастичным обменным взаимодействием, заставляет отдельные спины быть сонаправленными, таким образом препятствуя дефазировке и соответственно затуханию коллективной прецессии 2ДЭС с общей для всех электронов ларморовской частотой.

Было показано, что при фиксированной температуре отклонение фактора заполнения от величины, соответствующей квантово-холловскому ферромагнетику, приводит к спиновой деполяризации 2ДЭС и сопровождается скачкообразным уменьшением спиновой жесткости. В представленной работе мы на примере квантовохолловского ферромагнетика исследуем, как ведет себя спиновая жесткость в условиях спиновой деполяризации, вызванной увеличением электронной температуры. Оказывается, даже когда 2ДЭС сильно деполяризована (спиновая поляризация мало отличается от одночастичной), локальная спиновая жесткость 2ДЭС остается столь же большой, что и в спин-поляризованном состоянии, тем самым подразумевается существование спин-деполяризованной, но в то же самое время сильно спин-коррелированной новой электронной фазы. 


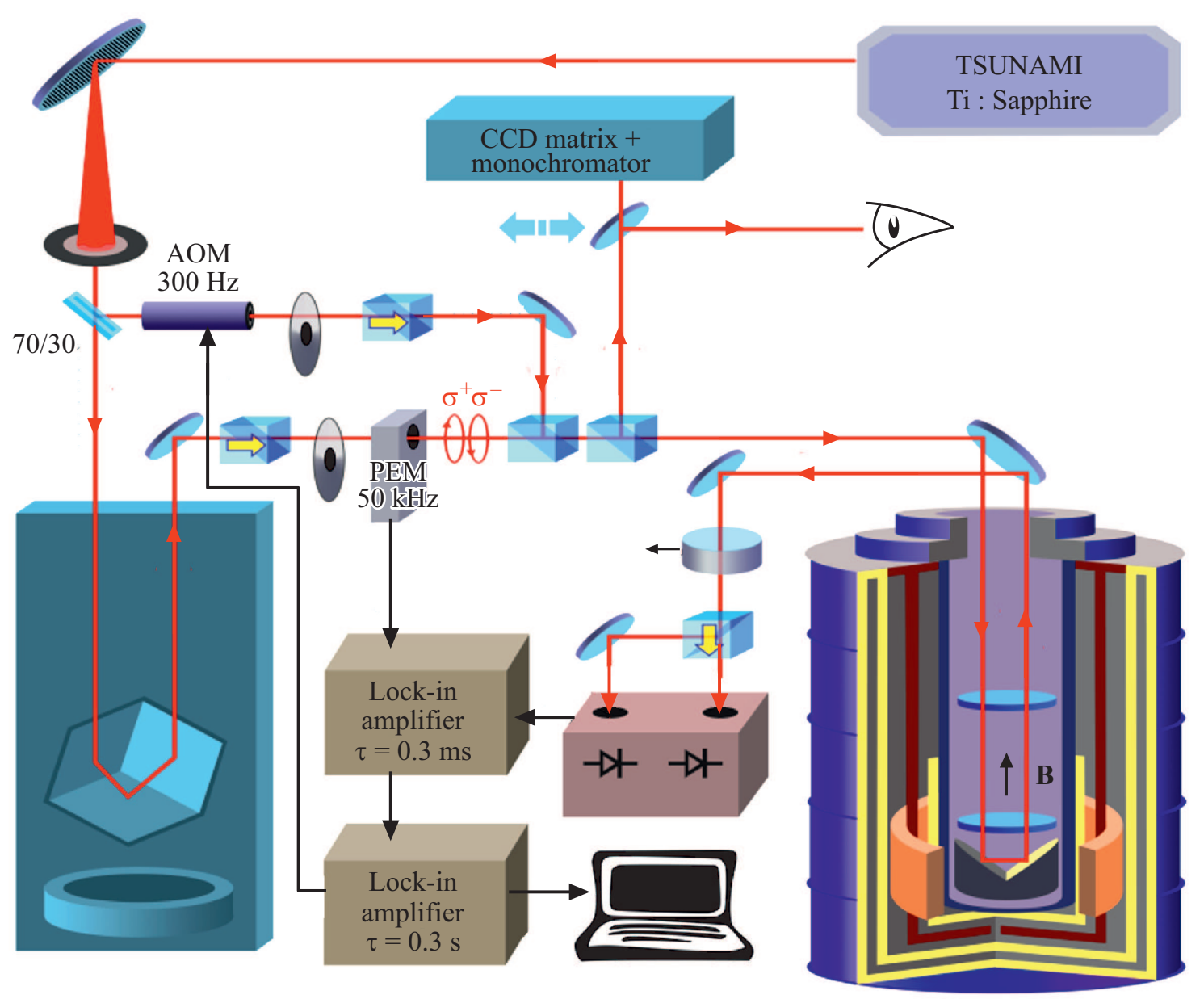

Рис. 1. Схема экспериментальной установки для измерения время-разрешенного магнитооптического вращения Керра.

\section{2. Исследуемые образцы и экспериментальная техника}

Исследовались высококачественные гетероструктуры $\mathrm{GaAs} / \mathrm{AlGaAs}$ с одиночной квантовой ямой шириной 18 нм, содержащей высокоподвижный двумерный (2D) электронный газ (транспортная подвижность $\left.\mu \approx 5 \cdot 10^{6} \mathrm{~cm}^{2} / \mathrm{B} \cdot \mathrm{c}\right)$ с темновой концентрацией электронов $n_{s} \approx 0.6 \cdot 10^{11} \mathrm{~cm}^{-2}$. Спиновая динамика двумерного электронного газа изучалась с помощью метода время-разрешенного спинового эффекта вращения Керра (рис. 1). Подробное описание установки можно найти в работе [2]. С помощью циркулярно-поляризованного импульса накачки в исследуемом образце создавалась спиновая ориентация двумерных электронов вблизи основного состояния электронной системы (нижний спиновый подуровень Ландау), которая регистрировалась по углу поворота плоскости поляризации отраженного от образца линейно поляризованного пучка зондирования с помощью специального балансного фотодетектора. Измерения керровского сигнала проводились в спектрально вырожденном режиме, при котором длина волны накачивающего и зондирующего лазерных пучков сов- падала. Средняя мощность накачки составляла величину $\sim 1$ мВт, размер лазерного пятна был $\sim 30$ мкм. При регистрации сигнала керровского вращения использовалось двойное синхронное детектирование, позволявшее эффективно подавлять паразитный рассеянный лазерный свет от поверхности гетероструктуры. Для этого осуществлялась дополнительная (амплитудная) модуляция пробного пучка с некоторой частотой, и на этой частоте модуляции регистрировался сигнал керровского вращения. Проведение температурных измерений в магнитном поле осуществлялось в криостате со сверхпроводящим соленоидом (критическое поле 10.2 Тл), содержащим вставку для конденсации изотопа гелия 3Не. Конструкция вставки позволяла плавно менять температуру в диапазоне от 0.6 до $15 \mathrm{~K}$. Непосредственный держатель образца содержал оптическое окно, через которое заводилось лазерное фотовозбуждение внутрь криостата и собирался полезный сигнал. Исследуемый образец монтировался в держателе таким образом, что наклон плоскости квантовой ямы к направлению вектора магнитной индукции составлял $45^{\circ}$. Это позволяло проводить измерения при различных факторах заполнения, a наличие продольной компоненты магнитного поля 
приводило к когерентной ларморовской прецессии двумерных электронов спинового подуровня Ландау.

\section{3. Экспериментальные результаты и обсуждение}

Для исследования изменения спиновой жесткости 2ДЭС в окрестности фактора заполнения $v=1$ нами были измерены сигналы время-разрешенного керровского вращения при различных факторах заполнения и температурах электронной системы $T=0.6,4.2$ и $10 \mathrm{~K}$ (рис. 2). Время затухания спиновой прецессии не различается при $T=0.6$ и $4.2 \mathrm{~K}$, что подразумевает практически одинаковую спиновую жесткость 2ДЭС. Зависимости времен затухания от фактора заполнения также совпадают в этих двух случаях, за исключением дополнительного максимума на низкотемпературной зависимости, связанной с возбуждениями спин-текстурной жидкости [3]. Для сравнения показана зависимость времени затухания, измеренная при $10 \mathrm{~K}$, которая представляет собой слабую монотонную зависимость от фактора заполнения, достигающую максимума на факторе заполнения $v=1$, и константу, не зависящую от фактора заполнения при $v>1$.

Рост времени затухания при повышении температуры в окрестности фактора заполнения $v=1$ связан с уменьшением флуктуаций $g$-фактора электронов. При низкой температуре добавляется вклад пространственных флуктуаций эффективного магнитного поля, действующего на электроны, от неравновесной поляризации ядерных спинов. Эти флуктуации не только существенно укорачивают время затухания сигнала керровского вращения, но и приводят к появлению дополнительных пиков в фурье-образе сигнала керровского вращения, соответствующих дополнительному эффективному магнитному полю на уровне 0.1 Тл (подробнее это обсуждается в работе [2]). Таким образом, пространственные флуктуации $g$-фактора и эффективного магнитного поля, действующего на электроны со стороны ядерных спинов через контактное взаимодействие, усиливаются при понижении температуры, что приводит к более быстрому затуханию спиновой прецессии 2ДЭС.

Установлено, что спиновая жесткость на факторе заполнения $v=1$ выживает при столь высоких температуpax (до $5 \mathrm{~K}$ ), при которых 2ДЭС практически полностью деполяризована $(\sim 20 \%)$. Это означает, что холловский ферромагнетик в процессе нагрева перед тем, как превратится в парамагнетик, переходит через дополнительное промежуточное фазовое состояние, характеризующееся высокой спиновой жесткостью и большой корреляционной длиной спин-спиновых корреляций, сравнимых с аналогичными в холловском ферромагнитном состоянии, но с малой средней по образцу спиновой поляризацией. Сравнение спиновой жесткости в спинтекстурной жидкости и в новой фазе подразумевает, что новая фаза не может быть спин-текстурной жидкостью

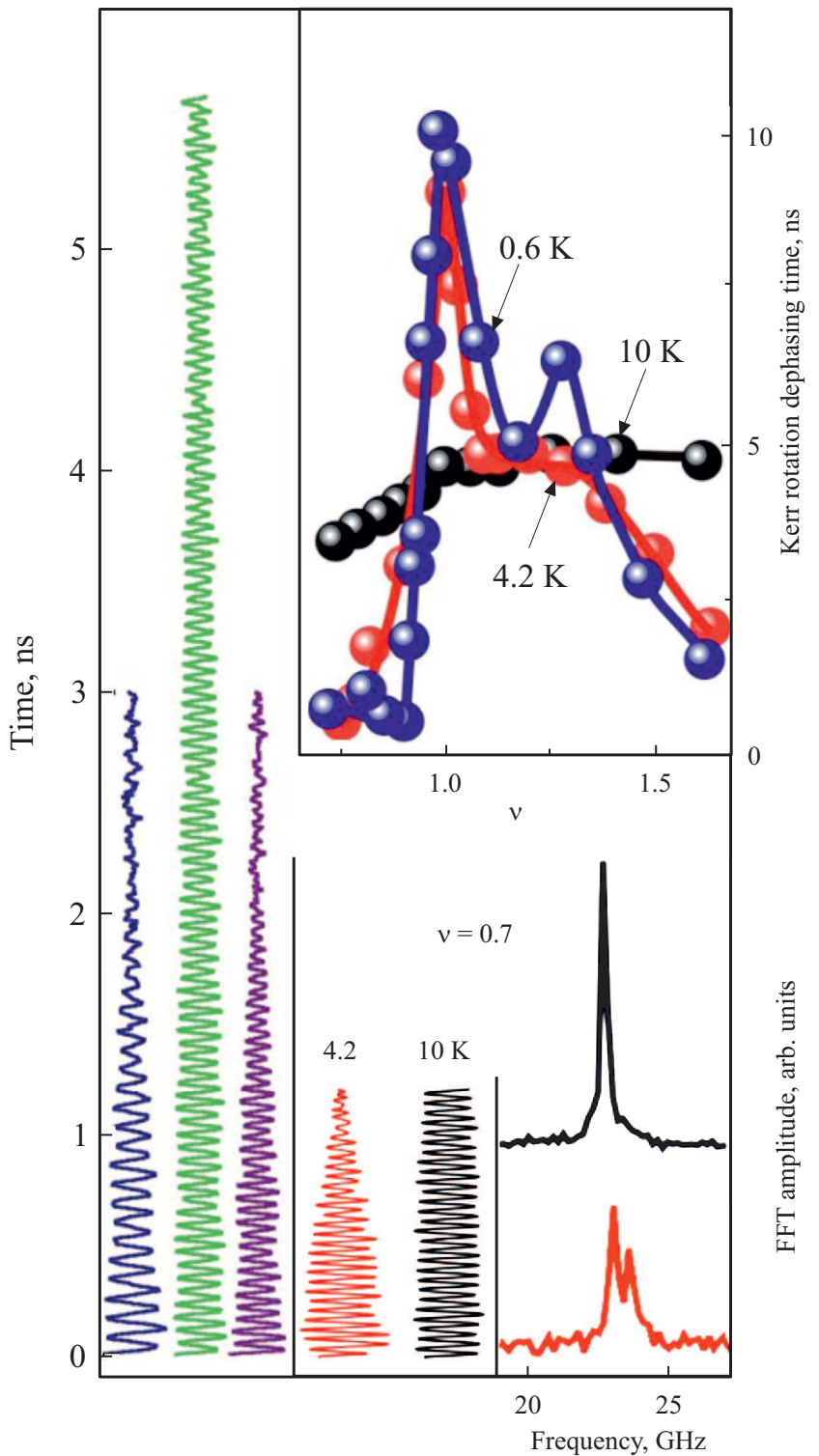

Рис. 2. Слева - временна́я динамика керровского сигнала при $T=0.6 \mathrm{~K}$ в зависимости от фактора заполнения. Вверху время спиновой дефазировки как функция фактора заполнения при $T=0.6,4.2$ и $10 \mathrm{~K}$. Внизу изображена динамика керровского сигнала, а также его фурье-преобразование (FFT) при $T=4.2$ и $10 \mathrm{~K}$, измеренные в состоянии с малой спиновой жесткостью при $v=0.7$.

(СТЖ). Указанным свойствам удовлетворяет система спинов, состоящая из текстур с большой корреляционной длиной (слабым изменением направления спина между соседними спинами). Такие текстуры не несут топологического заряда и число перевернутых спинов в них не квантовано, как в СТЖ (может оказаться существенно меньше единицы). Однако они обладают высокой спиновой жесткостью даже при значительной, как в $\mathrm{GaAs}$, зеемановской энергии.

Спиновая динамика электронов вблизи фактора заполнения $v=1$ имеет сложный характер и не описывается 


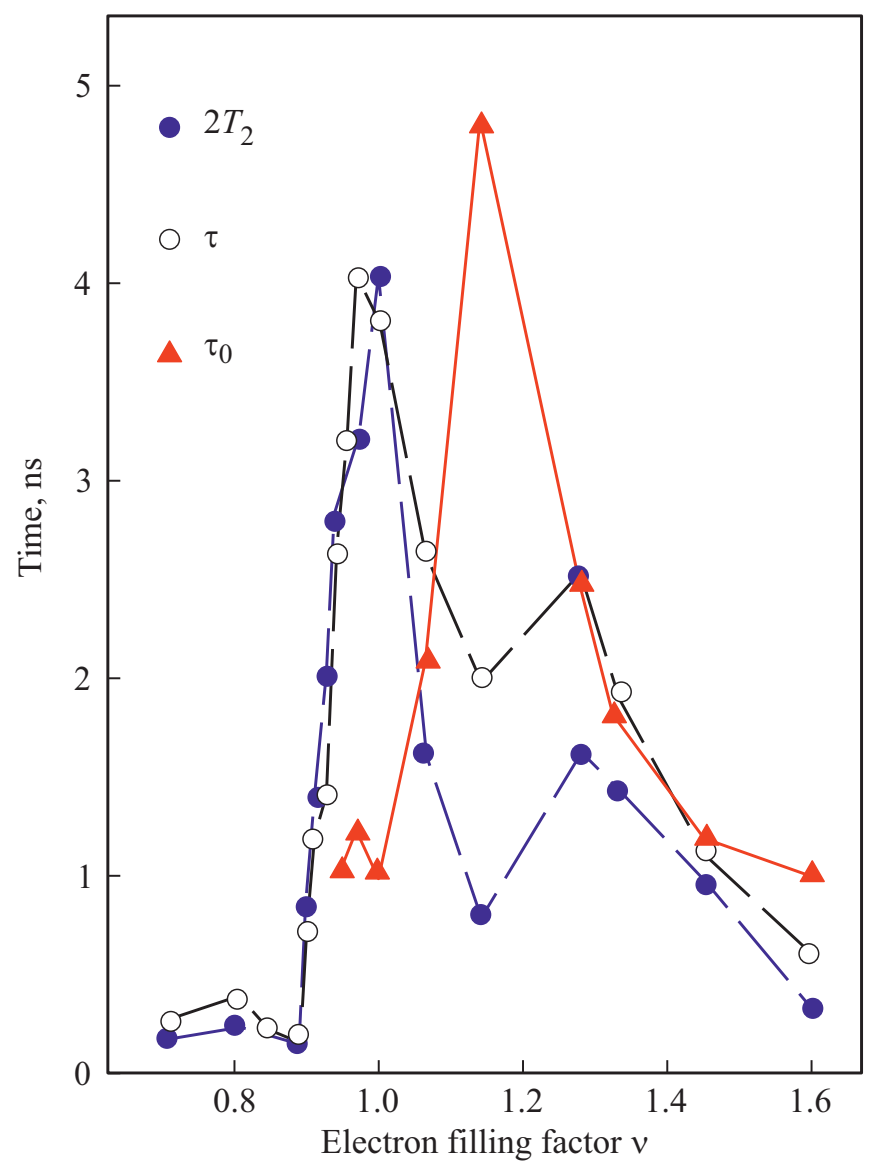

Рис. 3. Качественный вид зависимости характеристических времен $2 T_{2} \tau_{0}$ от фактора заполнения 2ДЭС (ошибка подгонки не превышает 1.5 раз от абсолютной величины). Для сравнения показано эмпирическое время и $\tau$, при котором амплитуда ларморовских осцилляций уменьшается в $e$ раз. Линии проведены для удобства восприятия.

простой экспоненциальной зависимостью. Время затухания когерентной спиновой прецессии 2ДЭС определяется пространственными флуктуациями эффективного $g$-фактора электронов и пространственными флуктуациями спина ядерной подсистемы полупроводника, в котором сформирована 2ДЭС (при гелиевых температурах вклад от ядерных спинов во флуктуирующее магнитное поле, испытываемое электронными спинами, становится доминирующим). Два эти вклада можно объединить в один, считая, что на электронные спины действует эффективное флуктуирующее магнитное поле, определяемое локальным эффективным $g$-фактором отдельного электрона и контактным взаимодействием этого электрона с локальным полем ядерной подсистемы. В пренебрежении обменным взаимодействием между электронами коллективная прецессия 2ДЭС распадается на локальные прецессии отдельных спинов с индивидуальной частотой Лармора, определяемой локальным значением флуктуирующего магнитного поля. В свою очередь спиновая жесткость, связанная с многочастич- ным обменным взаимодействием, заставляет отдельные спины быть сонаправленными, препятствуя дефазировке и соответственно затуханию коллективной прецессии 2ДЭС с общей для всех электронов ларморовской частотой. Таким образом, затухание амплитуды ларморовских осцилляций в сигнале керровского вращения от времени определяется статистикой флуктуаций эффективного магнитного поля $B$, действующего на электронные спины. Оказывается, что теоретическая разница между различными статистическими флуктуационными моделями невелика (она столь мала, что ее практически невозможно установить из существующих экспериментальных данных) [4]. Поэтому, без утраты общности, можно предположить наличие гауссова беспорядка в 2ДЭС: $\mathbf{G}(\mathbf{r})=\Delta_{g}^{2} \exp \left(-r^{2} / \Lambda^{2}\right)$. Здесь $\mathbf{G}(\mathbf{r})-$ корреляционная функция, описывающая флуктуации случайного потенциала, которая зависит от двух параметров $-\Delta_{g}$ и $\Lambda$, амплитуды и корреляционной длины флуктуаций соответственно. Воспользуемся выводами и обозначениями работы [4] для дальнейшего описания системы $\left(\Delta_{g}\right.$ выразим в единицах $\left.\mu_{\mathrm{B}} B\right)$. При выбранных предположениях амплитуда ларморовских осцилляций сигнала керровского вращения будет уменьшаться со временем как

$$
S_{+}(t)=S_{\text {perp }}(0) \exp \left(-i \varepsilon_{z} t\right)\left[1+\operatorname{Re} f_{a}(t)\right]
$$

где

$$
\begin{gathered}
\operatorname{Re} f_{a}=-\left(t / T_{2}\right)\left\{(2 / \pi) \arctan \left(2 t / t_{0}\right)\right. \\
\left.-\left(t_{0} / 2 \pi t\right) \ln \left[1+\left(2 t / \tau_{0}\right)^{2}\right]\right\}, \\
1 / T_{2}=\pi \tau_{0}\left(\Delta_{g} / 2\right)^{2}, \\
\tau_{0}=M_{x}\left(\Lambda / l_{\mathrm{B}}\right)^{2} .
\end{gathered}
$$

Здесь $l_{\mathrm{B}}-$ магнитная длина, $\varepsilon_{z}$ - ларморовская частота осцилляций, а величина $\hbar / M_{x}$ определяет спиновую жесткость системы (например, в холловском ферромагнетике она варьруется от 1 до 2 мэВ для стандартных 2ДЭС в квантовых ямах GaAs/AlGaAs). Зависимость амплитуды колебаний от времени не является экспоненциальной, как было принято считать. Характеристические времена $T_{2}$ и $\tau_{0}$ отвечают за линейный и нелинейный вклады в зависимость амплитуды ларморовских осцилляций сигнала керровского вращения. Из формулы (2) следует, что произведение двух характеристических времен является универсальной для любой спинполяризованной электронной системы величиной, не зависящей от спиновой жесткости 2ДЭС и определяемой исключительно одночастичным механизмом затухания керровского сигнала: $T_{2} \tau_{0}=(4 / \pi) \Delta_{g}^{-2}$.

Результат аппроксимации для характеристических времен $T_{2}$ и $\tau_{0}$ с использованием формул (1)-(3) показан на рис. 3 вместе со стандартной зависимостью уменьшения амплитуды ларморовских осцилляций сигнала керровского вращения в $e$ раз (время $\tau$ ). Видно, что эмпирическая и точная зависимости практически 
совпадают в широком диапазоне факторов заполнения в окрестности холловского ферромагнетика, что свидетельствует о близком к линейному уменьшении амплитуды.

Значительные нелинейности в исследуемой зависимости проявляются только в области формирования спинтекстурной жидкости [3] и точно на факторе заполнения $v=1$ (рис. 3). Здесь во временно́м поведении амплитуды затухания керровского сигнала сначала наблюдается участок в 1-2 нс с постоянным значением амплитуды, после чего происходит линейный спад, как это предсказано в работе [4]. Тем не менее произведение характеристических времен $2 T_{2}$ и $\tau_{0}$ во всей исследуемой области факторов заполнения остается практически неизменным, что позволяет оценить амплитуду флуктуаций эффективного магнитного поля $\left(\Delta_{g}\right)$. Эффективная амплитуда флуктуаций эффективного магнитного поля (ларморовской частоты), определенная из фурье-образа биений ларморовских осцилляций, составляет 0.003 мэВ, что вполне согласуется со средней амплитудой флуктуаций ларморовской частоты 0.0015, полученных аппроксимацией кривых затухания амплитуды ларморовских осцилляций сигнала керровского вращения. Корреляционная длина флуктуаций может быть оценена из известной энергии спиновой волны холловского ферромагнетика (1-2 мэВ в зависимости от электронной концентрации и ширины квантовой ямы) [4]. В нашем случае она составляет $\sim 300$ нм, что неплохо согласуется с длиной волны возбуждающего и регистрирующего лазерного излучения в объеме $\mathrm{GaAs}$, равной 225 нм (диэлектрическая проницаемость GaAs выбрана 12.9). Длина когерентности используемого лазера достаточно велика, поэтому даже при однородной засветке на образце возникают неоднородности интенсивности на масштабе длины волны света. Данные неоднородности влияют на спины ядер из-за взаимодействия двумерной электронной системы со спинами ядер. Поскольку поляризация ядер осуществляется лазерным пучком, полученная оценка выглядит вполне разумной при учете уменьшения диэлектрической проницаемости в плоскости квантовой ямы из-за близости к поверхности гетероструктуры.

\section{4. Заключение}

С помощью методики время-разрешенного керровского вращения исследована спин-деполяризованная электронная система на факторе заполнения $v=1$. Обнаружена новая сильно кореллированная фаза в спин-деполяризованной 2ДЭС, не имеющая очевидных аналогов в других состояниях 2ДЭС. Новая фаза характеризуется высокой степенью спиновой деполяризации и большой спиновой жесткостью, на порядок превышающей спиновую жесткость СТЖ в GaAs. Переход из нового спин-коррелированного состояния в состояние с низкой спиновой жесткостью, характерной для одночастичной электронной системы, происходит пороговым образом по температуре, что, возможно, указывает на связь новой фазы с топологическими фазами БерезинскогоКостерлица-Таулесса. Также экспериментально исследована структура сигнала керровского вращения от 2ДЭС в квантующем магнитном поле и показано, что в полном согласии с микроскопической теорией работы затухание коллективной спиновой прецессии 2ДЭС не имеет экспоненциального или линейного вида. Сделана попытка распространить результаты теоретической работы на область факторов заполнения, формально теорией не описываемую, и получить оценки на амплитуду флуктуаций магнитного поля, действующего на отдельные спины, а также на корреляционную длину этих флуктуаций. Экспериментальные оценки находятся в разумном согласии с ожидаемыми значениями. Экспериментальные результаты дают основание полагать, что методика времяразрешенного керровского вращения для коллективных спиновых состояний 2ДЭС может оказаться столь же информативной, что и магнитотранспортные методики для исследования коллективных зарядовых состояний 2ДЭС.

\section{Благодарности}

Авторы выражают благодарность С.М. Дикману за плодотворные обсуждения и замечания.

\section{Финансирование работы}

Работа выполнена при частичной поддержке Российского фонда фундаментальных исследований (грант № 18-02-01082).

\section{Конфликт интересов}

Авторы заявляют, что у них нет конфликта интересов.

\section{Список литературы}

[1] A.V. Larionov, L.V. Kulik, S. Dickmann, I.V. Kukushkin. Phys. Rev. B, 92, 165417 (2015).

[2] A.V. Larionov, E. Stepanets-Khussein, L.V. Kulik, V. Umansky, I.V. Kukushkin. Sci. Rep., 10, Article number: 2270 (2020).

[3] L.V. Kulik, A.S. Zhuravlev, V.E. Kirpichev, V.E. Bisti, I.V. Kukushkin. Phys. Rev. B, 87, 045316 (2013).

[4] S.M. Dickmann. J. Phys.: Condens. Matter, 32, 015603 (2020).

Редактор Л.В. Шаронова 